\title{
Répétabilité de la fertilité des canes Rouen et Pékin (Anas platyrynchos) en croisement interspécifique avec le Barbarie (Cairina moschata) par insémination artificielle
}

\author{
R. ROUVIER *, R. BABILÉ **, F. SALZMANN ***, \\ A. AUVERGNE **, B. POUJARDIEU * \\ avec la collaboration technique de F. Ruiz ${ }^{*}$, G. LATIL **, R. Combebiac *** \\ * I.N.R.A., Station d'Amélioration Génétique des Animaux, Centre de Recherches de Toulouse, \\ B.P. 27, F 31326 Castanet Tolosan Cedex \\ ** Ecole Nationale Supérieure Agronomique, Laboratoire de Zootechnie \\ et des Productions Animales, 145, av. de Muret, F 31076 Toulouse Cedex \\ *** SICA SEPALM Centre de Sélection des Palmipèdes Gras, Souprosse, F 40250 Mugron
}

\begin{abstract}
Résumé
Dans le but d’obtenir les répétabilités des taux de fertilité des canes communes en production de mulards par insémination artificielle, 2 expériences ont été conduites. Les inséminations avaient lieu 2 fois par semaine avec un mélange de sperme frais de 3 mâles Barbarie. Cinquante-trois canes Rouen, 83 et 62 canes Pékin ont été contrôlées individuellement pour tous leurs œufs pondus pour des durées respectives de $49,38,47$ jours et des intensités moyennes de ponte pendant ces périodes respectivement de 0,$83 ; 0,79$ et 0,91 . Les taux moyens d'œufs fécondés sont respectivement de 0,$69 ; 0,52$ et 0,61 . La moyenne générale est de 0,60 pour 7338 aufs mis à incuber. Les répétabilités du taux de fertilité dues à l'effet «cane » sont très significatives : 0,70 ; 0,89 et 0,88 respectivement. Il est confirmé que le taux d'œufs fécondés moyen dépend principalement de la maîtrise de la technique d'insémination artificielle. Il est conclu qu'il n'y a pas de barrière gamétique ou génétique entre les 2 espèces, mais qu'une barrière comportementale peut exister en accouplement naturel; que l'insémination artificielle pourrait être utilisée dans les programmes d'amélioration génétique de la cane commune pour améliorer la production de mulards, et que la transmission héréditaire de la fertilité de la cane commune en production d'embryons hybrides devra être étudiée.
\end{abstract}

Mots clés : Canard, mulard, croisement interspécifique, insémination artificielle, fertilité.

\section{Summary}

Fertility of Rouen and Pekin ducks (Anas platyrynchos) in interspecific crossbreeding with Muscovy (Cairina moschata) artificial insemination

In order to estimate the repeatability values for fertility rate of common duck for producing mule duck by artificial insemination, 2 experiments were made. The artificial inseminations occurred twice weekly with mixed fresh semen from 3 Muscovy drakes. The eggs laid by 53 Rouen ducks. 83 and 62 Pekin ducks were individually recorded for 49, 38 and 47 consecutive days 
respectively. The average egg laying intensities were $0.83 ; 0.79$ and 0.91 . The average proportions of fertile eggs among the incubated eggs were respectively $0.69 ; 0.52$ and 0.61 . The general mean is 0.60 for 7338 eggs set. Repeatability values are highly significant, varying from 0.70 to 0.89 . It is confirmed that the average fertility rate depends primarily on the mastering of the artificial insemination technique. It is concluded that there is no gametic or genetical barrier between the 2 species, but that a behavioral one may exist in natural matirg; that artificial insemination could be used in breeding programs to improve mule duck production from common duck and that hereditary transmission of fertility of the common duck for hybrid embryos has to be studied.

Key words : Duck, mule duck, inter-specific crossbreeding, artificial insemination, fertility.

\section{Introduction}

La mise en cuvre d'une méthode de sélection de la cane commune (Anas platyrynchos) en production de mulards obtenus en croisement avec le mâle Barbarie (Cairina moschata) nécessite une maîtrise de sa reproduction. En Europe, le taux d'œufs fécondés, en accouplement naturel, est faible (35 p. 100). A la suite des travaux expérimentaux précurseurs de ONISHI \& Kato (1955) ainsi que de Watanabe (1961), les éleveurs de Taïwan produisent les canetons mulards par insémination artificielle afin d'accroître le taux d'œufs fécondés (Rouvier et al., 1984).

Dans un programme d'amélioration des performances des populations de canes communes utilisées en France, la pratique de l'insémination artificielle pourrait donc permettre d'accroître l'efficacité de la méthode de sélection utilisée. Afin d'étudier cette question, il faut cependant d'abord vérifier la maîtrise obtenue en pratiquant cette technique sur nos populations de canes d'une part, d'autre part analyser les facteurs de variation génétiques et non génétiques des taux d'œufs fécondés et des taux de fertilité par cane. Dans ces buts, une première expérimentation a été conduite sur 2 souches de cane de types Rouen et Pékin. Les valeurs moyennes des taux d'œufs fécondés par insémination artificielle avec la semence de Barbarie et les répétabilités des taux de fertilité des canes ont été obtenues. L'objet de cette étude est de présenter et discuter ces résultats originaux.

\section{Matériel et méthodes}

\section{A. Matériel biologique}

Expérience 1. Les contrôles individuels portent sur les œufs pondus par 53 canes Rouen, sur 7 semaines de ponte du 13/04/85 au 31/05/85, au Domaine de Monlon de l'Ecole Nationale Supérieure Agronomique de Toulouse (ENSAT), après une période pré-expérimentale qui durait depuis le 22/02/85. Les canes, élevées au sol dans des parquets de 4 , étaient enfermées chaque soir dans des cages individuelles disposées dans ces parquets et dans lesquelles elles trouvaient de l'eau potable et de l'aliment à volonté. Le ramassage et l'identification des œufs se faisaient en début de matinée avant l'insémination des canes suivie de la mise dans les parquets. Ces inséminations artificielles étaient réalisées 2 fois par semaine, les lundi et jeudi. La mise en 
incubateur, hebdomadaire, avait lieu le vendredi. Le mirage des œufs, 10 jours plus tard, permettait de déterminer s'ils étaient observés fécondés ou non à ce stade.

\section{TABLEAU 1}

Durée des contrôles, nombre de canes contrôlées, taux moyens d'aufs fécondés et intensités moyennes de ponte des canes Rouen et Pékin.

Duration of recording in days, number of ducks, average fecundity rate of eggs and average egg laying intensities of Rouen and Pekin ducks.

\begin{tabular}{|c|c|c|c|}
\hline & \multirow{2}{*}{ Expérience 1} & \multicolumn{2}{|c|}{ Expérience 2} \\
\hline & & Essai 1 & Essai 2 \\
\hline Type génétique des canes & Rouen & Pékin & Pékin \\
\hline Durée des contrôles en jours & 49 & 38 & 47 \\
\hline Nombre de canes $\ldots \ldots$ & 53 & 83 & 62 \\
\hline Taux moyens d'œufs fécondés & 0,69 & 0,52 & 0,61 \\
\hline Intensités moyennes de ponte & 0,83 & 0,79 & 0,91 \\
\hline Semaine du pic de ponte & $24 / 03$ au $30 / 03$ & $17 / 03$ au $23 / 03$ & $28 / 07$ au $3 / 08$ \\
\hline
\end{tabular}

Expérience 2. Celle-ci a été conduite par la SICA SEPALM à Souprosse (Landes) avec une souche de canes de type Pékin. Deux essais sont rapportés. L'essai 1 concerne 83 canes contrôlées du $9 / 05 / 85$ au $15 / 06 / 85$ après une période pré-expérimentale qui a commencé le 21/03/85. L'essai 2 porte sur 62 canes contrôlées du 07/07/85 au 22/08/ 85. L'élevage des canes en reproduction en cages métalliques individuelles permettait le contrôle de l'identité des aufs. Les canes disposaient à volonté d'eau potable et d'aliment complet. Les inséminations artificielles avaient lieu en début de matinée après le ramassage des œufs, comme dans l'expérience 1. La périodicité des intervalles entre 2 inséminations successives a varié dans l'essai 1. Dans l'essai 2, les inséminations étaient réalisées systématiquement les mardi et vendredi. Les œufs mis en incubation toutes les 2 semaines étaient mirés 10 jours plus tard pour observer leur état de fécondation.

\section{B. Technique d'insémination artificielle}

La collecte du sperme des mâles Barbarie se faisait suivant la technique décrite par TAN (1980). Un mélange des éjaculats de 3 mâles récoltés dans une ampoule de verre servait immédiatement pour l'insémination des canes.

Selon Watanabe (1961), Huang \& TaI (communications personnelles), la semence fraîche doit être déposée au niveau de la jonction utérovaginale (sans la franchir), après avoir dépassé avec la pipette d'insémination le coude du tube vaginal. Dans ce but, l'on a procédé de 2 façons différentes dans les deux expériences : avec retournement du vagin, selon la technique utilisée par le Centre de Recherches sur le canard de Taïwan, dans l'expérience 1, sans retournement du vagin, selon la technique déjà décrite par Watanabe (1961) dans l'expérience 2. Les doses de sperme inséminées étaient de l'ordre de $0,05 \mathrm{cc}$. 


\section{Méthode statistique d'analyse}

Les taux d'œufs fécondés par cane ont été transformés en Arcsin $\sqrt{ }$ selon Bartlett (1947) en vue de leur analyse de variance, ou calcul de corrélations. Les propriétés de cette transformation, démontrées par Bogyo \& BEcker (1965) pour des calculs d'héritabilités de caractères «tout ou rien " ont été utilisées pour obtenir les répétabilités "cane" par décomposition de la variance entre canes. Si l'on écrit $y_{i}$ le taux d'œufs fécondés d'une cane i comme une somme de 3 statistiques indépendantes,

$$
y_{i}=p+m_{i}+b_{i}
$$

p étant la probabilité dans la population qu'un œuf mis en incubation soit observé fécondé au moment du mirage,

$m_{i}$ étant l'effet propre à la cane $\mathbf{i}$ mesuré en déviation par rapport à $p$, les $m_{i}$ étant indépendants entre eux, de variances $\sigma^{2} \mathrm{~m}$, d'espérances nulles,

$b_{i}$ étant l'effet aléatoire binômial mesuré en déviation par rapport à $p+m_{i}$, après la transformation Arcsin $\sqrt{y_{i}}$, si les angles sont exprimés en radians, l'on a :

$$
\mathrm{E}\left(\mathrm{b}_{\mathrm{i}}^{2}\right)=0,25 / \mathrm{r}_{\mathrm{i}}
$$

$r_{i}$ étant le nombre d'œufs mis en incubation de la cane $i$.

L'espérance mathématique de la variance entre canes du taux d'œufs fécondés exprimé en variable transformée s'écrit :

$E\left(s^{2}\right)=\sigma_{m}^{2}+\frac{1}{n} \sum_{i=1}^{n} \frac{0,25}{r_{i}}$

n étant le nombre de canes.

L'on estime donc $\sigma_{\mathrm{m}}^{2}$ par $\hat{\sigma}_{\mathrm{m}}^{2}$

$\mathrm{s}^{2}=\hat{\sigma}_{\mathrm{m}}^{2}+\frac{1}{\mathrm{n}} \sum_{\mathrm{i}=1}^{\mathrm{n}} \frac{0,25}{\mathrm{r}_{\mathrm{i}}}$

La répétabilité cane $\rho$ se calcule par:

$$
\rho=\frac{\hat{\sigma}_{\mathrm{m}}^{2}}{\mathrm{~s}^{2}}
$$

Cette répétabilité est une corrélation intra-classe. Sa signification statistique par rapport à 0 a été testée selon FiSHER (1958).

Les taux d'œufs fécondés moyen suivant le nombre de jours écoulés depuis la dernière insémination artificielle fécondante ont été calculés. Leur homogénéité a été testée par un test de $x^{2}$ pour mettre en évidence l'effet nombre de jours. 


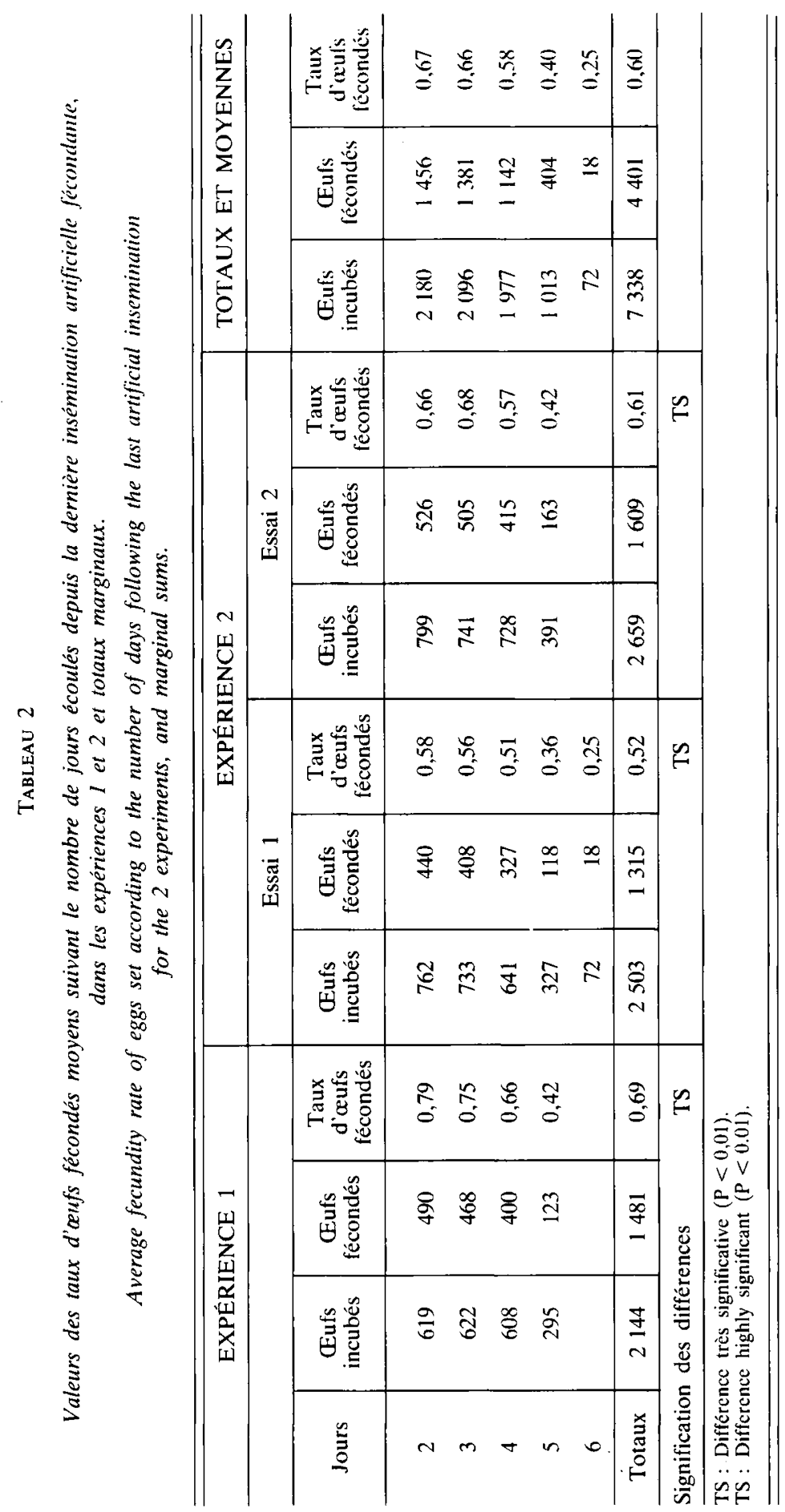




\section{Calculs et résultats}

Le tableau 1 indique, dans chacun des 3 cas, la durée des contrôles, le nombre de canes contrôlées, les taux moyens d'œufs fécondés, les intensités moyennes de ponte sur la période de contrôle, la date du pic de ponte.

Le tableau 2 indique les valeurs des taux d'œufs fécondés moyens suivant le nombre de jours écoulés depuis la dernière insémination artificielle fécondante, dans chacun des 3 cas et en moyennes générales. La moyenne générale du taux d'œufs fécondés est de 0,60 pour un total de 7338 œufs mis en incubation. Les taux d'œufs fécondés moyens observés dans les 3 cas, qui sont respectivement de 0,$69 ; 0,52$ et 0,61 , diffèrent significativement entre eux.

Ces taux d'ceufs fécondés moyens, calculés en fonction du nombre de jours depuis la dernière insémination artificielle fécondante, sont, en moyenne générale, du jour 2 au jour 6 , de $0.67 ; 0.66 ; 0,58 ; 0.40$ et 0.25 . La même tendance de diminution s'observe pour chacun des 3 cas séparément, pour lesquels les différences entre nombre de jours sont significatives. Cet effet «nombre de jours » n'existe pas entre les jours 2 et 3 (sauf dans l'expérience 1 où la différence est significative). Dans les 3 cas, l'on observe la même diminution significative du taux d'œufs fécondés à partir du $4^{\mathbf{c}}$ jour après la dernière insémination fécondante, avec une accentuation de cette décroissance le $5^{\mathrm{c}}$ jour.

Le tableau 3 donne les variances phénotypiques, les composantes « canes » de la variance, les coefficients de variation des taux de fertilité des canes et les coefficients de répétabilité.

Le coefficient de variation du taux de fertilité des canes est plus faible dans l'expérience $1(0,15)$ que dans l'expérience $2: 0,35$ et 0,22 respectivement dans les essais 1 et 2 . Les valeurs trouvées pour la répétabilité de la fertilité des canes sont fortes et très significativement $(\mathrm{P}<0,01)$ supérieures à $0: 0,70^{* *} ; 0,89^{* *}$ et $0,88^{* *}$, respectivement dans les 3 cas.

\section{Tableau 3}

Variance phénotvpique $\left(s^{2}\right)$, composante "cane » de la variance $\left(\hat{\sigma}_{m}^{2}\right)$, coefficient de variation et répétabilité du taux de fertilité des canes Rouen et Pékin, pour les données transformées en Arcsin $\sqrt{ }$.

Phenotypic variance $\left(s^{2}\right)$, duck component of variance $\left(\hat{\sigma}_{m}^{2}\right)$, coefficient of variation and repeatability

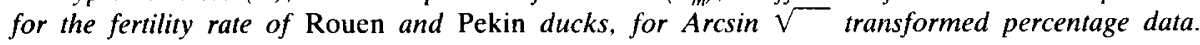

\begin{tabular}{|c|c|c|c|}
\hline & \multirow{2}{*}{ Expérience 1} & \multicolumn{2}{|c|}{ Expérience 2} \\
\hline & & Essai 1 & Essai 2 \\
\hline Type génétique des canes & Rouen & Pékin & Pékin \\
\hline Variance phénotypique $\mathrm{s}^{2}$ & 0,022 & 0,079 & 0,039 \\
\hline Composante de la variance $\hat{\sigma}_{\mathrm{m}}^{2} \ldots$ & 0,015 & 0,070 & 0,034 \\
\hline Coefficient de variation $\ldots \ldots \ldots$ & 0,15 & 0,35 & 0,22 \\
\hline Répétabilité $\ldots \ldots \ldots \ldots \ldots \ldots \ldots$ & $0,70^{* *}$ & $0,89 * *$ & $0,88^{* *}$ \\
\hline
\end{tabular}

** Valeur très significativement supéricure à $0(\mathrm{P}<0,01)$. 


\section{Diseussion}

Watanabe (1961) donne des premiers résultats en production d'embryons hybrides issus de l'insémination de la cane commune avec de la semence de Barbarie. Mais son étude porte sur un trop petit nombre de canes inséminées (5) et d'œufs pondus (25) pour être significative. Les seules autres données publiées auxquelles nous pouvons comparer nos résultats sont celles de HuANG \& CHOw (1974) reprises dans Rouvier et al. (1984). Ces auteurs ont d'abord déterminé qu'il n'y a pas de différence de fertilisation des œufs suivant que l'on insémine 0,01 ou $0,025 \mathrm{~cm}^{3}$ de semence fraîche mélangée de Barbarie. Sur 5 lots de 38 canes communes chinoises (Tsaiya) contrôlées pendant 114 jours, les intervalles d'insémination avec de la semence de Barbarie étaient de $2,3,4,5$ et 10 jours. Les taux moyens d'œufs fécondés étaient respectivement de 0,$81 ; 0,71 ; 0,62 ; 0,46$ et 0,22 .

Nos résultats ne sont pas exactement comparables. La dose de sperme inséminée est de l'ordre de $0,05 \mathrm{~cm}^{3}$ par cane. Les canes du même groupe sont inséminées à des intervalles de temps successifs de durées données. Nos résultats confirment cependant que la diminution du taux d'œufs fécondés moyen intervient à partir du $4^{\mathrm{e}}$ jour après l'insémination et $s^{\prime}$ accentue le $5^{\mathfrak{c}}$ jour. Il vaudrait donc mieux inséminer avec des intervalles successifs de 2 ou 3 jours, pour obtenir des taux moyens d'œufs fécondés plus élevés.

Les résultats obtenus montrent également que la maîtrise de l'insémination artificielle est un facteur important pour l'obtention des embryons hybrides, confirmant ainsi les hypothèses de Rouvier et al. (1984). En effet, le taux d'œufs fécondés moyen obtenu avec un matériel génétique différent de celui de Taïwan est cependant élevé : 0,60 pour 0,70 à Taïwan en fermes (et 0,80 dans la meilleure ferme). Cela peut s'expliquer par une difficulté technique de dépôt de la semence dans le site de l'insémination, sur notre matériel expérimental. D'après Watanabe (1961), le pénis du mâle peut, en accouplement naturel, s'insérer par le cloaque et l'orifice vaginal jusqu'au sphincter du vagin (décrit par BoBR et al., 1964).

Selon Huang (communication personnelle), des inséminations qui ont pour site le vagin profond conduisent aux taux d'œufs fécondés les plus élevés. L'anatomie particulière du vagin de la cane dont la tunique interne présente de nombreux replis (Lignereux, communication personnelle) explique une difficulté technique qui pourrait être plus ou moins grande suivant les animaux, pour réaliser l'insémination en ce site. Lorsqu'on dépose la semence dans ce site, l'on peut obtenir des taux d'œufs fécondés très élevés (jusqu'à 0,89 pour des pontes des $2^{\mathrm{c}}$ et $3^{\mathrm{c}}$ jours après l'insémination, obtenu sur 2 jours dans l'expérience 1).

Il s'ensuit que l'hypothèse d'incompatibilité gamétique ou génétique qui a pu être avancée par certains auteurs pour expliquer un faible taux de fécondation dans les accouplements mâles Cairina $\times$ femelle Anas, n'est pas vérifiée. De plus, dans l'expérience 2 où l'on a pu mesurer l'éclosabilité des œufs fécondés ainsi que celle des œufs de canes contemporaines conduites en accouplements naturels, cela dans le même couvoir, l'éclosabilité moyenne est la même dans les 2 cas : 0,82 et 0,84 respectivement, alors que le taux d'œufs fécondés est très inférieur en saillie naturelle. Cela écarte l'éventualité d'une incompatibilité entre les 2 espèces au cours du développement embryonnaire de l'hybride. Des taux de fécondation plus faibles souvent observés en accouplements naturels pourraient être liés au comportement sexuel : faible fréquence 
des accouplements (mais ceci serait à vérifier) ou difficulté de réalisation de ceux-ci dans le cas de dimorphisme sexuel en poids important. En appui à la première hypothèse, Gvaryahu et al. (1984) indiquent qu'ils ont observé que lorsque plus de 10 mâles Barbarie sont dans le même parc, ils sont occupés par des combats territoriaux, une activité homosexuelle, et s'occupent moins des canes Pékin. Huang \& CHow (1974) ont établi le mode d'utilisation de l'insémination artificielle afin de lever une barrière à l'accouplement naturel du canard de Barbarie et de la cane Tsaiya due à la grande différence de taille corporelle.

La répétabilité du taux de fertilité des canes n'a jamais été mise en évidence jusqu'à présent, à notre connaissance. Bien que la comparaison entre les expériences 1 et 2 ne puisse pas être interprétée, l'on peut noter qu'elle est plus faible dans l'expérience 1 sur canes Rouen que dans l'expérience 2 sur canes Pékin. Le coefficient de variation est également plus faible, alors que le taux moyen d'œufs fécondés est plus élevé. Dans les 3 cas considérés, le coefficient de variation varie en sens inverse du taux moyen d'œufs fécondés. Il serait donc un indicateur de la maîtrise de la technique d'insémination utilisée et de l'adaptation moyenne des animaux à la technique. Lorsque la maîtrise est plus grande, la part de variation due aux effets des canes sur leur taux de fertilité est plus faible relativement à la variation totale. Cette remarque montre qu'il sera difficile de conclure d'après la répétabilité seule et qu'une étude génétique complète sera nécessaire.

Il reste que la répétabilité des effets « cane » sur la fertilité est forte. En mesurant le taux de fertilité des canes sur 6 semaines de ponte, l'on peut obtenir une valeur prédictive suffisamment précise de l'effet dû à la cane, quelle que soit la population animale. Ces valeurs élevées des répétabilités pourraient s'expliquer par des raisons pathologiques (pour les très faibles taux de fécondation), anatomiques ou génétiques. Les facteurs pathologiques éventuels des canes à très faible taux d'œufs fécondés n'ont pas été recherchés. Sur le $2^{\mathrm{c}}$ point, d'après ce qui a été discuté sur la mise en place de la semence, l'on pourrait penser à une plus ou moins bonne adaptation de la cane à l'insémination artificielle selon la structure anatomique de son vagin. Pour discuter les facteurs génétiques, il faudra étudier la transmission héréditaire de la fertilité des canes.

\section{Conclusion}

Les fortes valeurs des répétabilités indiquent la présence d'un effet " cane " comme réponse à la technique d'insémination artificielle utilisée pour le taux de fertilité. Un facteur important pourrait être la plus ou moins grande adaptation de la cane, au point de vue anatomique, à la technique utilisée pour la mise en place de la semence au site choisi pour l'insémination. La répétabilité est une limite supérieure de l'héritabilité. Les valeurs trouvées peuvent dépendre de la maitrise technique de l'insémination artificielle. La transmission héréditaire de la fertilité des canes en production d'embryons hybrides mulards devra donc être étudiée ainsi que les relations génétiques avec l'éclosabilité. A ce point de vue, les résultats discutés indiquent que les hypothèses d'incompatibilité gamétique et génétique dans l'hybridation des 2 espèces de canards ne sont pas vérifiées. L'insémination artificielle pourrait donc être utilisée comme technique de 
reproduction pour le test de la valeur génétique des canes dans un programme de sélection pour améliorer les performances des mulards (prolificité des canes et caractères de production des mulards).

Reçu le 14 mars 1986.

Accepté le 3 juillet 1986.

\section{Remerciements}

Nous remercions Marie-Madeleine Mialon pour son aide dans la saisie des données du Centre de Sélection des Palmipèdes Gras de Souprosse et dans la réalisation des calculs.

\section{Références bibliographiques}

Bartlett M.S., 1947. The use of transformations. Biometrics, 3, 39-52.

Bobr L.W., Lorenz F.W., Ogasawara F.X., 1964. Residence sites of spermatozoa in fowl oviducks. J. Reprod. Fert., 8, 39-47.

Bogyo T.P., BecKer W.A., 1965. Estimates of heritability from transformed percentage sib data with unequal subclass numbers. Biometrics, 21, 1001-1007.

Fisher R.A., 1958. Statistical Methods for Research workers. 13th ed., 351 pp., Oliver and Boyd, Edinburgh.

Gvaryahu G., Robinzon B., Meltzer A., Perek M., Snapir N., 1984. Artificial insemination and natural mating in the crossbreeding of the Muscovy drake and the Pekin duck. Poult. Sci., 63, 386-387.

Huang H.H., Chow T.C., 1974. Artificial insemination in mule duck production. Proc. XV World Poult. Congress, New-Orleans, August 11-16, 1974, 261-262, Proceeding and abstracts, Washington USA.

ONISH N., Kato Y., 1955. On the mule duck production by means of a new artificial insemination technic. Bull. Nat. Inst. Agric. Sci. Series $G, \mathrm{n}^{\circ} 11,17-31$.

Rouvier R., TAI J.J.L., TAI C., 1984. L'insémination artificielle des canes communes pour la production de mulard à Taïwan. La situation actuelle. In : Insémination artificielle et amélioration génétique: bilan et perspectives critiques; Les Colloques de l'INRA, $\mathbf{n}^{\circ} 29,360-367$.

TAN N.S., 1980. The training of drakes for semen collection. Ann. Zootech., 29, 93-102.

Watanabe M., 1961. Experimental studies on the artificial insemination of domestic ducks with special reference to the production of mule ducks. J. Fac. Fish. Anim. Husb. Hiroshima Univ., 3, 439-478. 